\title{
The infrared counterpart of the X-ray burster KS 1731-260
}

\author{
R. P. Mignani ${ }^{1}$, S. Chaty ${ }^{2}$, I. F. Mirabel ${ }^{3,4}$, and S. Mereghetti ${ }^{5}$ \\ 1 ESO, Karl Schwarzschild Str. 2, 85748 Garching bei München, Germany \\ 2 The Open University, Department of Physics and Astronomy Walton Hall, Milton Keynes, MK7 6AA, UK \\ 3 Service d'Astrophysique / CEA, CE-Saclay, 91191 Gif-sur-Yvette, France \\ 4 Instituto de Astronomía y Física del Espacio/CONICET. cc5, 1428 Bs As, Argentina \\ 5 Istituto di Astrofisica Spaziale e Fisica Cosmica CNR, Sezione di Milano "G.Occhialini", via Bassini 15, \\ 20133 Milano, Italy
}

Received 8 March 2002 / Accepted 26 April 2002

\begin{abstract}
We present $J K^{\prime}$ infrared images of the X-ray transient KS 1731-260, obtained in 1997 and in 1998 with IRAC2b at the ESO/MPI 2.2-m telescope at La Silla. Using as a reference the recent Chandra position, we confirm the identification of the X-ray source with the previously proposed counterpart (Wijnands et al. 2001b), for which we measure $J=17.32 \pm 0.2$ and $K^{\prime}=16.36 \pm 0.18$. The source was entering a low X-ray state at the epoch of our observations, and the accretion disk was still dominating the infrared flux. Indeed, when compared with the only published magnitudes in the $J$-band (Orosz et al. 2001), obtained with the source in quiescence, our photometry confirms the fading of the counterpart during the decay of the RXTE/ASM lightcurve.
\end{abstract}

Key words. X-ray binaries, KS 1731-260

\section{Introduction}

KS 1731-260 was discovered in the galactic plane region $\left(l=1.073^{\circ} ; b=3.653^{\circ}\right)$ by the TTM imaging spectrometer aboard the MIR-KVANT observatory (Sunyaev et al. 1989). Soon after the discovery, several type I X-ray bursts were detected by TTM (Sunyaev et al. 1990), thus establishing that the source is an LMXB with an accreting neutron star. A long term monitoring of the source with the RXTE (Muno et al. 2000) has revealed a possible correlation between the properties of the bursts and the source spectral state. Coherent oscillations at $524 \mathrm{~Hz}$, possibly associated to the neutron star rotation period, have been discovered by the RXTE/PCA during several X-ray bursts (Smith et al. 1997) as well as kHz QPOs in the persistent emission (Wijnands \& van der Klis 1997). The measurement of the photospheric radius expansion during a burst by RXTE (Smith et al. 1997; Muno et al. 2000) allowed to estimate the source distance $(7 \mathrm{kpc})$. Recently, a superburst has been discovered by the WFC aboard BeppoSAX

Send offprint requests to: R. P. Mignani,

e-mail: rmignani@eso.org

* Based on observations collected at the European Southern Observatory, La Silla, Chile, under proposal numbers 59.D0719, 61.D-0542.

$\star \star \mathrm{RPM}$ and SC contributed equally to this work.
(Kuulkers et al. 2002). $\mathrm{A} \approx 38$ days periodicity in the $\mathrm{X}$-ray flux was recently discovered by Revnivtsev \& Sunyaev (2001). While KS 1731-260 is a nearly persistent source in soft X-rays (Barret et al. 1998), it is highly variable in the hard X-ray domain, where it exhibits flux variations occuring on time scales of days to weeks (see, e.g., Kuulkers et al. 2002). However, the X-ray activity of the source underwent a gradual decrease and since one year it has entered into a quiescent state (Wijnands et al. 2001a; Wijnands et al. 2002).

The identification of the companion star to KS 1731260 has been for a long time hampered both by the high reddening along the galactic plane and by the lack of an accurate X-ray position. The two candidate optical counterparts proposed by Cherepashuk et al. (1994) were excluded by later infrared observations (Barret et al. 1998). Only recently, the precise X-ray position provided by Chandra observations (Revnivtsev \& Sunyaev 2002) has allowed to identify an optical counterpart in the $I$ band (Wijnands et al. 2001b), although without providing its flux measurement. Soon after, the counterpart has been observed in the $J$ band by Orosz et al. (2001).

Here, we present and independent confirmation of the source identification based on the analysis of images of the KS 1731-260 field obtained back in 1997 and in 1998 with the IRAC2b camera at the ESO/MPI $2.2 \mathrm{~m}$ telescope in 
Table 1. Observations of KS 1731-260 obtained with the IRAC2b camera at the ESO/MPI $2.2 \mathrm{~m}$ telescope. The observing dates, the exposure times, the number of exposures per passband and the seeing conditions are listed.

\begin{tabular}{cccc}
\hline \hline Date & $J$ & $K^{\prime}$ & seeing \\
\hline 1997.07 .19 & $15 \times 1 \mathrm{~m}$ & $15 \times 1 \mathrm{~m}$ & $1^{\prime \prime} .2$ \\
1998.07 .06 & $9 \times 1 \mathrm{~m}$ & $9 \times 1 \mathrm{~m}$ & $0^{\prime \prime} 8$ \\
1998.07 .07 & $9 \times 1 \mathrm{~m}$ & $9 \times 1 \mathrm{~m}$ & $1^{\prime \prime} 0$ \\
\hline
\end{tabular}

the $J$ and $K^{\prime}$ bands. The observations are described in Sect. 2 and the results in Sect. 3.

\section{Observations and data reduction}

The observations of the KS 1731-260 field were carried out in two runs on July 19th 1997 and July 6th/7th 1998 at the European Southern Observatory (ESO) in La Silla (Chile) using the ESO/MPI $2.2 \mathrm{~m}$ telescope. Images of the field were acquired in the infrared with the IRAC2b camera through the $J(\lambda=1.247 \mu \mathrm{m}, \Delta \lambda=0.290 \mu \mathrm{m})$ and $K^{\prime}(\lambda=2.2 \mu \mathrm{m}, \Delta \lambda=0.32 \mu \mathrm{m})$ passbands. The IRAC2b camera was mounted at the $\mathrm{F} / 35$ infrared adapter of the telescope. The instrument was a Rockwell $256 \times 256$ pixels $\mathrm{Hg}$ :Cd:Te NICMOS 3 large format infrared array detector. It was used with the lens $\mathrm{C}$, providing an image scale of 0 ". 49/pixel and a field of $136 \times 136 \operatorname{arcsec}^{2}$. The observing strategy was identical for the two observing runs, with the source being observed in both $J$ and $K^{\prime}$ through a sequence of repeated exposures of $1 \mathrm{~min}$ each. After each image of the target field, an image offset by 30 arcsec was taken to allow for sky subtraction. The total integration time per passband was 15 min for the 1997 run, while two integrations of 9 min each per passband were collected in 1998 to monitor for short term variability. The log of the observations is summarized in Table 1. The typical seeing conditions for the observations were varying between 0.7 and $1^{\prime \prime} 2$.

After sky subtraction, the images have been corrected for the instrumental effects, by removal of the bias, dark current and flatfielding, using standard procedures available in IRAF. The final images have then been registered and combined. Photometric calibration was performed using observations of 7 standard stars, with some of them repeatedly observed during the night to allow for accurate zero-points determination.

In order to register the accurate Chandra coordinates of the source $-\alpha(J 2000)=17^{\mathrm{h}} 34^{\mathrm{m}} 13.34 \pm 0.04$; $\delta(J 2000)=-26^{\circ} 05^{\prime} 18^{\prime \prime} .7 \pm 00^{\prime \prime} 6$ (Revnivtsev \& Sunyaev 2002) - on our images, we needed a precise astrometric solution. Thus, the image astrometry has been computed using as a reference the positions of stars selected from the recently released Guide Star Catalogue II (GSC-II), which has an intrinsic absolute astrometric accuracy of $\approx 0$ !! 3 per coordinate (McLean et al. 2002). A total of 25 GSC-II
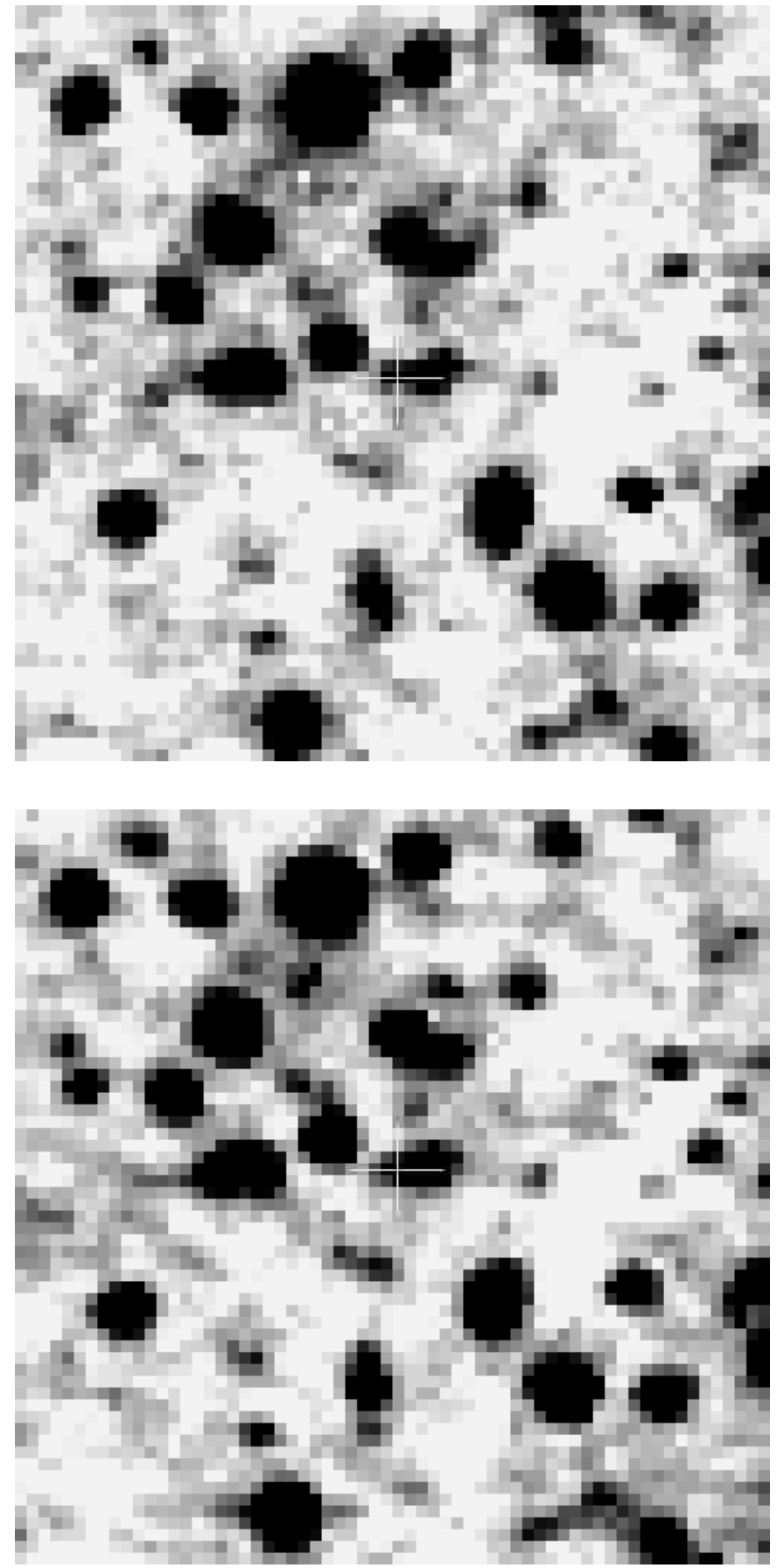

Fig. 1. (top) $30^{\prime \prime} \times 30^{\prime \prime}$ cutout of the combined $J$ band image of the KS 1731-260 field obtained on July 6th 1998 with the IRAC2b camera at the ESO/MPI $2.2 \mathrm{~m}$ telescope. North to the top, East to the left. The cross marks the position of the X-ray source according to the Chandra localization (Revnivtsev \& Sunyaev 2002). The size of the cross arms corresponds to 3 times the overall uncertainty on the source position as given from the combination of the intrinsic coordinates accuracy and our astrometric solution (see text). (bottom) Same, but for the $K^{\prime}$ band image.

objects have been identified in one of the averaged $J$-band images and used as astrometric calibrators. The pixel coordinates of the reference stars have been computed by a 

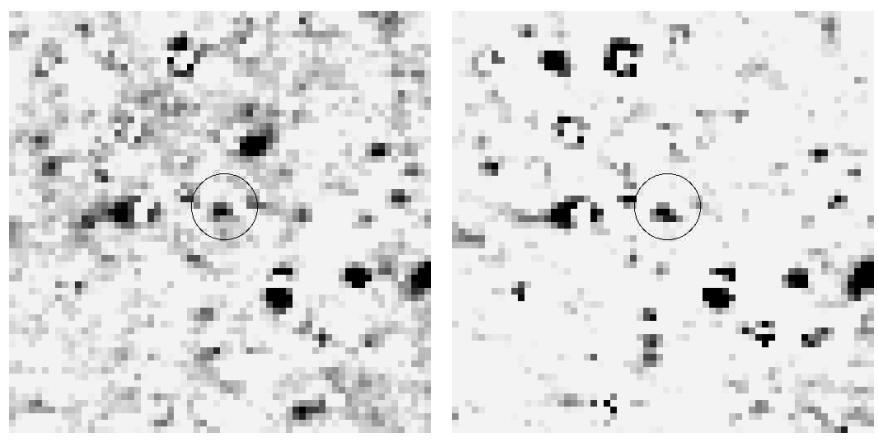

Fig. 2. $15^{\prime \prime} \times 15^{\prime \prime}$ zoom around the source position after star subtraction for the $J$ (left) and $K^{\prime}$ (right) images. The KS 1731-260 counterpart is marked by a circle.

two-dimensional Gaussian fitting procedure and transformation from pixel to sky coordinates was then computed using the program ASTROM (Wallace 1992), yielding an rms of $\sim 0$ "' 18 in both Right Ascension and Declination, which we assume representative of the accuracy of our astrometric solution. The final uncertainty to be attached to the source position is $\sim 0$.' 65 in both coordinates and takes into account the errors of the source coordinates $(0$. " 6$)$, the rms error of our astrometric fit $\left(\sim 00^{\prime \prime} 18\right)$ and the propagation of the intrinsic absolute errors on the GSC-II coordinates $\left(\sim 0^{\prime \prime} 12\right)$.

\section{Results}

Figure 1 show $30^{\prime \prime} \times 30^{\prime \prime}$ cutouts of the July 6 th $1998 J$ and $K^{\prime}$ images centered around the computed source position and taken as a reference because of the better seeing conditions (see Table 1). The source position, marked with a cross, clearly coincides with the fainter of a doublet of stars, only partially resolved in our images. The westernmost of the two is star H of Barret et al. (1998), while the fainter star is unambiguously identified as the counterpart originally proposed by Wijnands et al. (2001). Thus, our astrometry provides a clear and independent confirmation of the source identification.

In order to obtain reliable magnitude measurements for the counterpart, we have applied a star subtraction alghoritm to remove star $\mathrm{H}$ as well as other nearby objects. This step was applied by computing a model PSF using the IRAF/DAOPHOT package and was iterated to minimize the residuals after PSF subtraction.

Figure 2 show a $15^{\prime \prime} \times 15^{\prime \prime}$ zoom of the $J$ and $K^{\prime}$ band images, respectively, after PSF subtraction. The circle indicates the KS 1731-260 counterpart. The source magnitudes have been computed to be $J=17.32 \pm 0.2$ and $K^{\prime}=16.36 \pm 0.18$. To have an independent assessment of our photometric calibration in the $J$ band, we recomputed the magnitudes of the 13 field objects listed in Table 1 of Barret et al. (1998), using the IRAF/DAOPHOT package for the photometry in crowded fields. We found a very good agreement, with deviations within 0.01 mag.

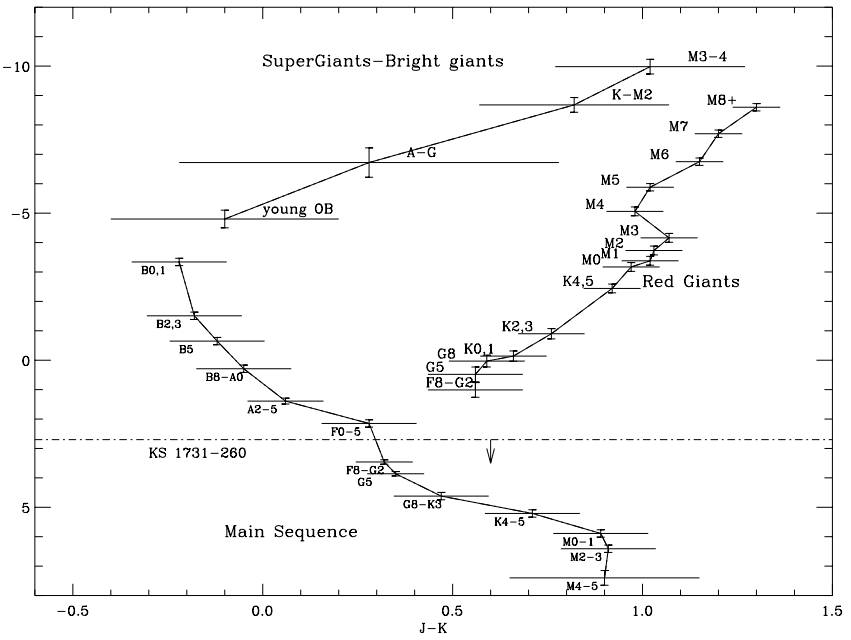

Fig. 3. $J, J-K^{\prime}$ absolute CMD diagram computed for template stars (Ruelas-Mayorga 1991). The position of the companion star to KS 1731-260 in the diagram is anywhere below the dot-dashed line, corresponding to the absolute magnitude derived from the photometry of Orosz et al. (2000) assuming a distance upper limit of $d=7.0 \mathrm{kpc}$ (Muno et al. 2001) and an absorption $A_{V} \simeq 6$ derived from Wijnands et al. (2002).

We have used our complete dataset to carry out absolute and relative photometry to search for short and long term variations in the flux of the counterpart, to be correlated with the evolution of the X-ray lightcurve. Unfortunately, the poorer quality of the second 1998 observation and of the 1997 one did not allow us to obtain significant detections and reliable flux measurements for the counterpart. However, compared with the value $(J=$ $18.62 \pm 0.21)$ measured by Orosz et al. (2001) when the $\mathrm{X}$-ray source was in its low-state, our $J$ band magnitude clearly confirms that the counterpart is indeed variable.

\section{Discussion}

The comparison between the photometry of Orosz et al. (2001) demonstrates that, although the RXTE/ASM lightcurve shows that KS 1731-260 was entering a low $\mathrm{X}$-ray state, the contribution of the accretion disc to the observed infrared emission was still dominant at the time of our 1998 observations. Thus, the nature of the companion star can not be constrained by our photometry without arbitrary assumption on the evolution of the $J-K^{\prime}$ of the counterpart during the transition of the source to the low X-ray state. However, we note that if we take the $J$-band magnitude of Orosz et al. (2001) as representative of the intrinsic flux of the companion star, for the most recent distance estimate of $d \leq 7.0 \mathrm{kpc}$ (Muno et al. 2000) and the $A_{V} \simeq 6$ obtained from the spectral fits to the combined Chandra/XMM-Newton data (Wijnands et al. 2002 ) we derive an upper limit on the absolute magnitude $M_{J} \approx 2.7 \pm 0.8$, where the quoted error also accounts for the uncertainties on the absorption estimates. This value is plotted in Fig. 3 together with the theoretical sequences computed from Ruelas-Mayorga (1991). As it is seen, the 
diagram suggests that if the companion is on the main sequence it must be of an intermediate spectral type, probably later than F. On the other hand, if the companion has evolved off the main sequence, we can likely exclude it is a Red Giant.

Infrared spectroscopy with the VLT will certainly allow to obtain a more precise classification of the companion star.

Acknowledgements. We thanks the anonymous referee for her/his useful comments to the manuscript. RPM and SC gratefully acknowledge the ESO Office for Science, which funded a visit of SC at ESO (Garching), during which this work was finalized. SC also acknowledges support from grant F/00-180/A from the Leverhulme Trust. The Guide Star Catalogue - II is produced by the Space Telescope Science Institute in collaboration with the Osservatorio Astronomico di Torino. Space Telescope Science Institute is operated by the Association of Universities for Research in Astronomy, for the National Aeronautics and Space Administration under contract NAS5-26555. Additional support is provided by the Italian Council for Research in Astronomy, European Southern Observatory, Space Telescope European Coordinating Facility, the International GEMINI project and the European Space Agency Astrophysics Division.

\section{References}

Barret, D., Motch, C., \& Predehl, P. 1998, A\&A, 329, 965 Kuulkers, E., in 't Zand, J. J. M., van Kerkwijk, M. H., et al. 2002, A\&A, 382, 583

Muno, M. P., Fox, D. W., \& Morgan, E. H. 2000, ApJ, 542, 1016

Orosz, J. A., BailYn, C. D., \& Whitman, K. 2001, ATEL \#75

Revnivtsev, M. G., \& Sunyaev, R. A. 2001, A\&A, submitted [astro-ph/0108323]

Revnivtsev, M. G., \& Sunyaev, R. A. 2002, AstL, 28, 19

Ruelas-Mayorga, R. A. 1991, Rev. Mex. Astron. Astrofis., 22, 27

Smith, D. A., Morgan, E. H., \& Bradt, H. 1997, ApJ, 479, L137

Sunyaev, R. A., \& the Kwant Team 1989, IAUC, 4839

Sunyaev, R. A., Gilfanov, M., Churazov, E., et al. 1990, Sov. Astr. Lett., 16(1), 59

Wallace, P. T. 1992, Stalink User Note, 5.13

Wijnands, R., \& van der Klis, M. 1997, ApJ, 482, L65

Wijnands, R., Groot, P. J., Miller, J. J., Markwardt, C., Lewin, W. H. G., \& van der Klis, M. 2001a, ATEL \#72

Wijnands, R., Miller, J. J., Markwardt, C., Lewin, W. H. G., \& van der Klis, M. 2001b, ApJ, 560, L159

Wijnands, R., Guainazzi, M., van der Klis, M., \& Mendez, M. 2002, ApJ, submitted [astro-ph/0202398] 